\title{
Papillary hidradenoma: immunohistochemical analysis of steroid receptor profile with a focus on apocrine differentiation
}

\author{
Annamaria Offidani, Anna Campanati
}

\begin{abstract}
Aim-To make a quantitative evaluation by image analysis of oestrogen receptors, progesterone receptors, and androgen receptors in papillary hidradenomas and anogenital sweat glands.

Methods-20 papillary hidradenomas and the anogenital sweat glands detected in surgical specimens selected from 10 vulvectomies for squamous carcinoma, eight haemorrhoidectomies, and one anal polypectomy, all from female patients, were investigated by the avidinstreptavidin peroxidase testing system.

Results- $90 \%$ of papillary hidradenomas and almost all the anogenital sweat glands showed immunoreactivity for oestrogen receptor and, more weakly, for progesterone receptor, with immunolabelled nuclear area ranging from $10 \%$ to $90 \%$. Conversely conventional sweat glands did not show any nuclear staining. Overexpression of androgen receptors occurred in $20 \%$ of papillary hidradenomas, with nuclear staining strictly bordering papillary epithelium with apocrine differentiation. There was no immunoreactivity for androgen receptors in anogenital sweat glands.
\end{abstract}

Conclusions-Oestrogen and progesterone receptors seem to represent reliable markers for differentiating between anogenital sweat glands and conventional sweat glands, and a further link to explain why papillary hidradenomas occur almost exclusively in the female anogenital region. Positivity for oestrogen/progesterone receptors suggests that epithelia either of anogenital sweat glands or of papillary hidradenomas are controlled by ovarian steroid hormones. Androgen receptor nuclear staining of the epithelium with apocrine differentiation in vulvar papillary hidradenoma strengthens its homology with breast duct papilloma.

(f Clin Pathol 1999;52:829-832)

Dermatology Clinic, University of Ancona, Ospedale Umberto 1, Largo Cappelli I, 60123 Ancona, Italy

A Offidani

A Campanati

Correspondence to:

Professor Offidani

Accepted for publication 6 July 1999
Keywords: steroid receptors; anogenital sweat glands; papillary hidradenomas; apocrine differentiation

Oestrogen and progesterone receptors have recently been carefully investigated in both benign and malignant eccrine sweat gland tumours, primarily to identify a marker for differentiating breast cancer metastases from eccrine neoplasms. Syringoma, chondroid syringoma, eccrine hidradenoma, eccrine carcinoma, and microcystic adnexal carcinoma have sometimes been shown to express oestrogen receptors and progesterone receptors. ${ }^{1-3}$ However, there have been few reports on the steroid receptor profile of papillary hidradenoma, which has unique clinicopathological features as it develops only in postpuberal and postmenopausal women and is localised almost exclusively to vulvar, perineal, and perianal skin. ${ }^{45}$ Moreover in recent years several histological studies of the female anogenital region have carefully examined a new variant of cutaneous glands, the so called "anogenital sweat glands," which are considered the most likely source of papillary hidradenomas. ${ }^{6}$ Our objective in this immunohistochemical study was to make a quantitative evaluation of oestrogen receptors, progesterone receptors, and androgen receptors. By focusing attention on the apocrine differentiation of papillary epithelium, we aimed to provide further immunohistochemical evidence for the supposed histogenetic linkage of papillary hidradenoma with anogenital sweat glands, as well its steroid hormone dependence.

\section{Methods}

The patient data and specimens used in this study were retrieved by AC from the files of the department of pathology and the dermatology clinic of the University of Ancona from 1986 to 1997. The investigation was performed on 19 excisional biopsies, including 20 papillary hidradenomas. In addition, anogenital sweat glands were analysed in the specimens from 10 vulvectomies performed for squamous carcinoma, eight hemorrhoidectomies, and one anal polypectomy, all from female patients. Sections from archival formalin fixed, paraffin embedded tissues were stained using conventional procedures, and by the alcian blue ( $\mathrm{pH}$ 2.5) and periodic acid-Schiff (PAS) methods for mucins. Serial sections were also incubated with a panel of primary antibodies, all known to react with formaldehyde fixed, paraffin embedded tissue, against the following: low molecular weight keratins (CAM 5.2; Becton Dickinson), high molecular weight keratins (AE 1-3; Diagnostic Products Corporation), carcinoembrionic antigen (anti-CEA; Ylem), human milk fat globule protein (anti-HMFG 1 and 2; Oxoid), S 100 (anti-S protein; Dakopatts), actin (HHF 35; Dakopatts), vimentin (anti-vimentin; Dakopatts), lysozyme (antilysozyme; Dakopatts), EMA (anti-EMA; Dakopatts), and receptors for oestrogen (oestrogen receptor ID5; BioGenex), progesterone (PGR-1A6; BioGenex), and androgen 
(F39.4.1; BioGenex). The avidin-streptavidinperoxidase testing system was employed. All preparations of papillary hidradenomas were briefly counterstained with ethyl green and those of anogenital sweat glands with haematoxylin. Non-immune mouse and rabbit sera were substituted as negative controls. Appropriate positive controls were run concurrently for all the antibodies tested. Tissue samples from breast and prostate cancers, known to bear oestrogen/progesterone receptors and androgen receptors, acted as external controls. Stromal vimentin staining (fibroblasts, endothelium) was used as an internal control of antigenicity as its epitope shows a vulnerability to fixation or processing comparable to that shown by steroid receptor antigens. ${ }^{7}$ The oestrogen and progesterone receptors were quantified on slides with the Cell Analysis System 200 instrument (CAS 200). ${ }^{8}$ Information concerning clinical presentation, treatment, and outcome was sought for all patients. Complete excision of hidradenomas was easily accomplished and postoperative course was uneventful.

\section{Results}

CLINICAL FEATURES

All patients were in postpuberal and postmenopausal age groups: the youngest was 26 years old and the oldest 92 (mean 51 years). One patient had two hidradenomas. Nine of the 19 patients were asymptomatic; in three, the tumour was a fortuitous finding in specimens from haemorrhoidectomies. Bleeding and ulcerated lesions had occurred in three patients. Four patients reported fluctuation in size of the tumor with the menstrual cycle. The clinical diagnosis of the lesion was correct in five cases; misdiagnoses included cysts in nine, haemorrhoids in three, and melanoma and carcinoma in one case each. Tumour sizes ranged from 0.3 $\mathrm{cm}$ to $4 \mathrm{~cm}$ (mean: $1.2 \mathrm{~cm}$ )

\section{HISTOPATHOLOGY}

All 20 tumours fulfilled the diagnostic criteria of papillary hidradenoma, as reported in standard textbooks of dermatopathology. ${ }^{9}{ }^{10}$ In five cases we detected areas of apocrine differentiation of papillary epithelium where lining cells appeared columnar, cuboidal, or flattened, depending entirely on the location of the lesion on papillary folds or in tension cysts. These cells were equipped with dome shaped, abundant, granular, and strongly eosinophilic cytoplasm and pale, globoid nuclei with one or two nucleoli. Anogenital sweat glands were selected according to the morphological description provided by van der Putte. ${ }^{6}$

IMMUNOHISTOCHEMISTRY

The epithelium of both papillary hidradenomas and anogenital sweat glands rested on a outer layer of myoepithelial cells that gave a strong reaction with antibodies directed against smooth muscle actin (HHF35), S100 protein, and, more weakly, CAM 5.2. The inner luminal layer of columnar cells, mainly with apical "snouts," expressed distinct reactivity for antibodies against low molecular weight kera- tin, human milk fat globules (HMFG), and lysozyme but not for those against carcinoembrionic antigen (CEA). The cells with apocrine differentiation were distinctly immunoreactive to epithelial membrane antigen, keratin, and lysozyme, but not to carcinoembrionic antigen.

\section{RECEPTORS}

Anogenital sweat glands reacted strongly with antibodies to oestrogen receptor and moderately to progesterone receptor, whereas there was no immunoreactivity to androgen receptor. Interestingly, in glands with an intermediate morphology encompassing anogenital sweat glands with "a small segment of typical eccrine epithelium", ${ }^{6}$ only nuclei of anogenital epithelium stained with oestrogen receptor and progesterone receptor antibodies.

Eighteen papillary hidradenomas (90\%) showed immunoreactivity for oestrogen/progesterone receptor which was scored according to per cent positive nuclear surface ${ }^{11}: 0$, negative (two cases); 1 , less than $1-10 \%$ positive (no cases); $2,1-10 \%$ positive (no cases); 3, 10-33\% positive (five cases); 4, 34-66\% positive (seven cases); $5,67-100 \%$ positive (sic cases). We chose a score of $10 \%$ as the cut off to include borderline positive cases in the positive category. The staining intensity was calculated on the basis of 1 , weak; 2, moderate; and 3 , intense nuclear staining. The oestrogen receptor score staining was 3 in all positive cases, whereas progesterone receptor staining score was 2 in 13 cases and 1 in five cases. There was no relation between staining degree and the age of the patients. No immunostaining was obtained for oestrogen and progesterone receptors in epithelium with apocrine differentiation; in contrast, it showed overexpression of androgen receptors as almost all the nuclei were immunolabelled. Two papillary hidradenomas did not show any staining for either steroid receptors or vimentin filaments.

\section{Discussion}

Although an unusual lesion, papillary hidradenoma represents the most common benign adnexal tumour of vulvar skin. ${ }^{12}$ The hypothesis that this tumour originates directly from apocrine sweat glands conflicts with its main clinicopathological features: it has not been reported in men, it does not develop in the axillary regions, and it does not appear to occur in black women. ${ }^{4}$ The common factor in these exceptions is the presence of high concentrations of apocrine sweat glands.

Several recent studies of the microscopic anatomy of the female anogenital region have examined in detail a new variant of cutaneous glands, the so called anogenital sweat glands, which are considered to be the most likely source of papillary hidradenomas ${ }^{6}$ (fig 1). In this immunohistochemical investigation, we evaluated oestrogen receptors, progesterone receptors, and androgen receptors in a large series of anogenital sweat glands in which the epithelial cells were distinctly reactive to oestrogen receptors (fig 2) and to a lesser degree to progesterone receptor, but not to androgen receptor. 


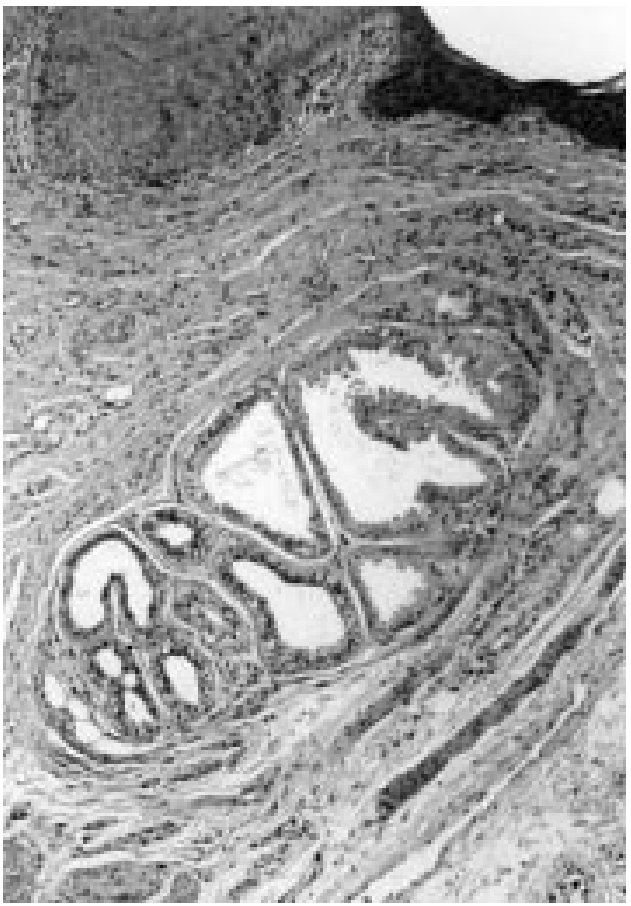

Figure 1 Anogenital sweat gland with characteristic localisation in vulvar sulcus interlabialis: coiled duct is lined by columnar cells with intraluminal snouts and apocrine type secretion. Haematoxylin and eosin stain.

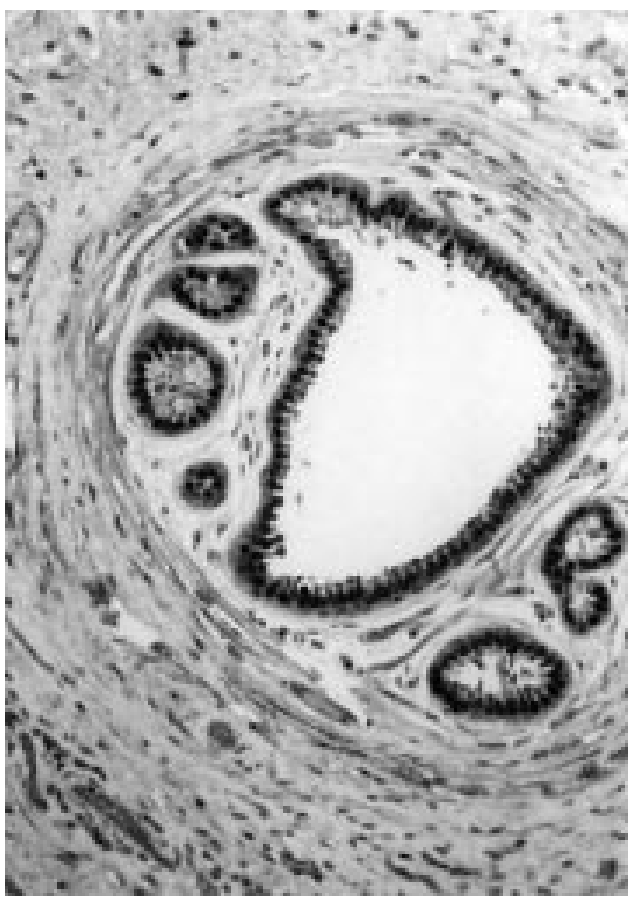

Figure 2 Anogenital sweat gland: immunohistochemical detection of oestrogen receptor on formalin fixed, paraffin embedded tissue. Nuclear area was strongly stained up to $95 \%$ with anti-oestrogen receptor antibody (ER-ID5). Immunoperoxidase staining counterstained with haematoxylin.

Interestingly, in anogenital sweat glands of intermediate type equipped with both "anogenital" and typical eccrine epithelium, only the former was immunoreactive to oestrogen and progesterone receptors. There was no correlation between number of nuclei expressing oestrogen/progesterone receptor and age of the patients. Typical eccrine and apocrine sweat glands included in specimens selected were devoid of either oestrogen receptors or progesterone receptors, while immunoreactivity for androgen receptor was detected in eccrine secretory coils and apocrine luminal cells. These results agree with those previously reported. ${ }^{13-15}$ Thus oestrogen and progesterone receptors seem reliable markers for differentiating female anogenital glands from conventional sweat glands.

This report, based on the quantitative evaluation by image analysis of oestrogen, progesterone, and androgen receptors in 20 papillary hidradenomas, is one of the most complete, as other reports have included fewer cases, ${ }^{16}$ or have involved testing for oestrogen receptor alone. ${ }^{2}$ Ninety per cent of papillary hidradenomas showed immunoreactivity for oestrogen receptor and more weakly for progesterone receptor (fig 3), and labelling ranged from $90 \%$ to $10 \%$ of nuclear area. We think that the negativity in two cases was caused by loss of antigenicity during fixation and processing rather than because of a true lack of steroid receptors.

Thus the presence of oestrogen and progesterone receptors tends to support the assumption that papillary hidradenoma is derived from anogenital epithelium expressing these receptors rather than from conventional apocrine glands.

In $20 \%$ of our papillary hidradenomas, immunoreactivity for androgen receptor was present but was strictly limited to luminal cells bearing the phenomenon of so called "apocrine differentiation" (fig 4), in the same way as in breast proliferative disorders, where it has been investigated in detail. ${ }^{17} 18$

This finding, in combination with the morphological features, underscores the homology between papillary hidradenoma and breast duct papilloma and supports the view that papillary hidradenoma originates from ectopic breast tissue, which may occur anywhere along the embryonic milk line. The vulva lies in this line and is rich in apocrine glands, modification of which may give rise to accessory well developed breast tissue. Nevertheless this occurrence is exceedingly rare, since only 35 cases had been documented in published reports up to $1988 .{ }^{19}$ We did not find any well formed breast tissue in our specimens, and papillary hidradenomas were mainly found in the neighbourhood of anogenital sweat glands. Moreover they were also located in perianal skin, which is considered to be out of the milk line and, on the average, they were of smaller size than ectopic breast tissue. In addition, no patients in our series described vulvar symptoms during pregnancy and lactation, which might be expected as ectopic breast tissue is capable of behaving in a similar way to the normal breast and responds to the hormonal influences. ${ }^{20}$ The presence of oestrogen receptors, and even more so of progesterone receptors, which are a phenotypical markers of hormonal action, ${ }^{21}{ }^{22}$ suggests that the luminal cells of anogenital sweat glands and papillary 


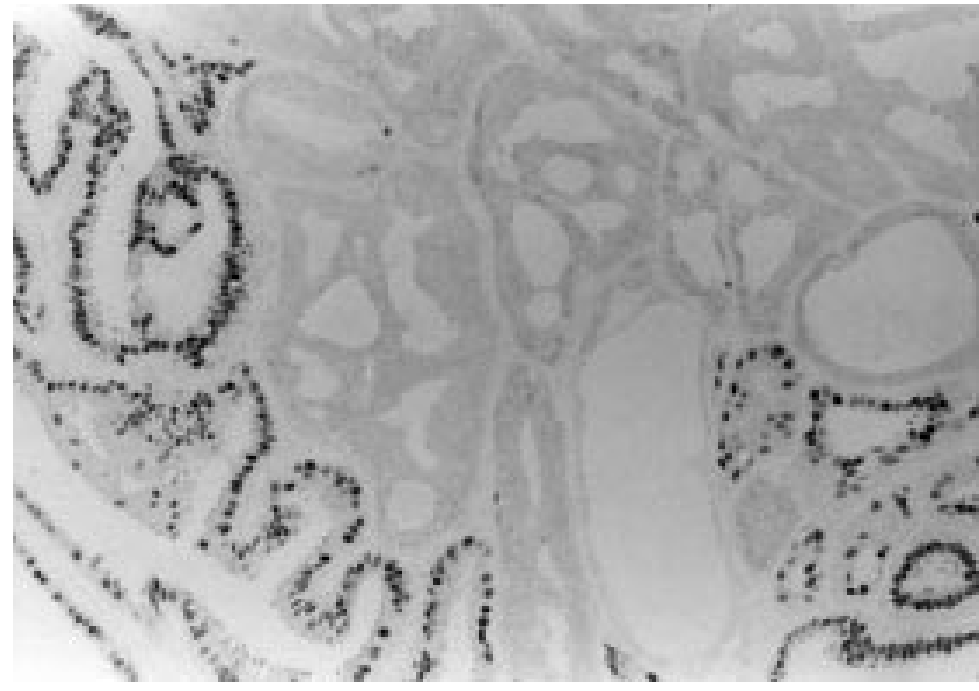

Figure 3 Papillary hidradenoma: immunohistochemical detection of progesterone receptor on formalin fixed, paraffin embedded tissue. Almost all nuclei lining papillary folds were stained with anti-progesterone receptor antibody (PGR-1A6). Conversely the cells with apocrine differentiation did not show any reactivity. Immunoperoxidase staining weakly counterstained with ethyl green.

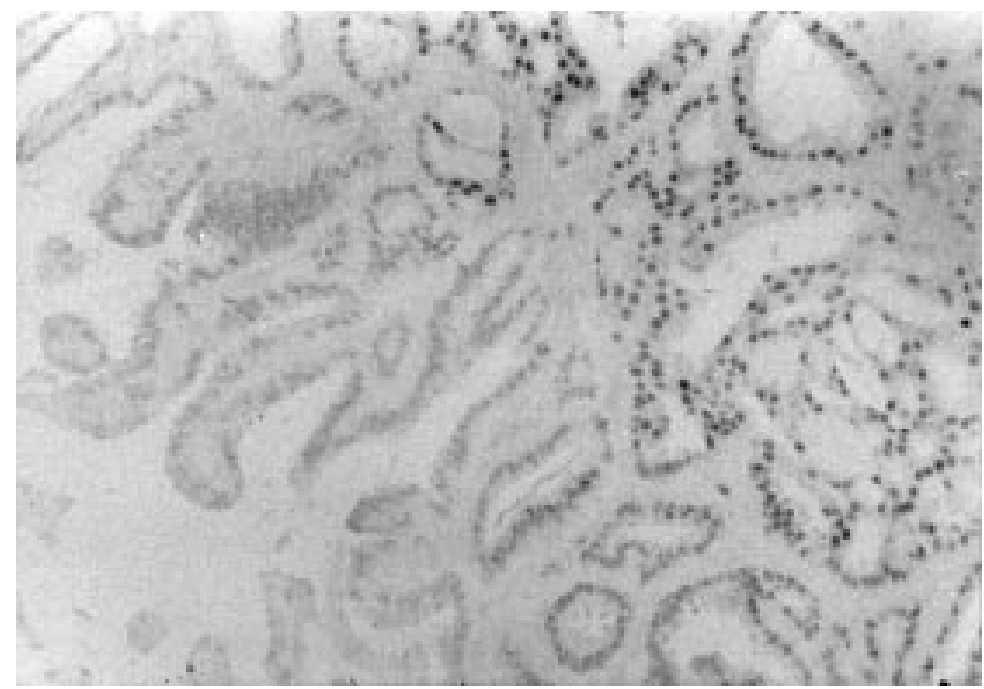

Figure 4 Papillary hidradenoma: immunohistochemical detection of androgen receptor on formalin fixed, paraffin embedded tissue. Only cells with apocrine differentiation stained for anti-AR antibody (F 39.4.1) while epithelium lining folds was negative.

Immunoperoxidase staining weakly counterstained with ethyl green.

hidradenomas are controlled by ovarian steroid hormones.

On the basis of this immunohistochemical analysis, we believe that oestrogen receptors and progesterone receptors can be useful additional markers for distinguishing between anogenital sweat glands and typical sweat glands, which is never easy on morphological grounds alone. In addition the presence of nuclear staining for oestrogen receptor/progesterone receptors in both anogenital sweat glands and papillary epithelium tends to support the assumption that papillary hidradenomas are derived from these glands, rather than directly from conventional apocrine sweat glands, the latter being devoid of oestrogen and progesterone receptors. Finally the coexpression of steroid receptors has enabled us to classify the papillary hidradenoma in the group of hormone related tumours.

1 Wallace ML, Longacre TA, Smoller BR. Estrogen and progesterone receptors and anti-gross cystic disease fluid protein 15 (BRST-2) fail to distinguish metastatic breast carcinoma from eccrine neoplasms. Mod Pathol 1995;8:897901.

2 Swanson PE, Mazoujian G, Mills SE, et al. Immunoreactivty for estrogen receptor protein in sweat gland tumors. $\mathrm{Am}$ f Surg Pathol 1991;15:835-41.

3 Wallace ML, Smoller BR. Progesterone receptor positivity upports hormonal control of syringoma. F Cutan Pathol 1995;22:442-5.

4 Woodworth H, Dockerty MB, Wilson RB, et al. Papillary hidradenoma of the vulva: a clinicopathologic study of 69 cases. Am f Obstet Gynecol 1971;110:501-8.

5 Meeker JH, Neubecker RD, Helwig EB. Hidradenoma papilliferum. Am f Clin Pathol 1962;37:182-95.

6 Van der Putte SCJ. Anogenital "sweat" glands. Histology and pathology of a gland that may mimic mammary glands. Am f Dermatopathol 1991;13:557-67.

7 Battifora H. Assessment of antigen damage in immunohistochemistry. The vimentin internal control. Am $\mathcal{F}$ Clin Pathol 1991;96:669-71.

8 Esteban JM, Kandalaft PL, Mehta P, et al. Improvements on the quantification of estrogen and progesterone receptors on paraffin-embedded tumors by image analysis. Am f Clin Pathol 1993;99:32-8.

9 McKee PH. Hidradenoma papilliferum. Pathology of the skin with clinical correlations, 2nd ed. London: Mosby-Wolfe, 1996:15.75-6.

10 Elder D, Elenitsas R, Ragsdaler BD. Hidradenoma papilliferum. In: Elder D, ed. Lever's histopathology of the skin, 8th ed. Philadelphia: Lippincot-Raven, 1997:770-1.

11 Elias JM, Cartun RA, England DM, et al. Interlaboratory comparison of estrogen receptor analysis in paraffin sections by a monoclonal antibody to estrophilin (H222). $\mathcal{F}$ Histotechnol 1993;16:57-63.

12 Michael H, Roth LW. Papillary hidradenomas. In: Wilkinson EJ ed. Pathology of the vulva and vagina. New York: Churchill Livingstone, 1987:31-2.

13 Lloveras B, Googe PB, Goldberg DE, et al. Estrogen receptors in skin appendage tumors and extramammary Paget's disease. Mod Pathol 1991;4:487-90.

14 Shikata N, Kurokawa I, Andachi H, et al. Expression of androgen receptors in skin appendage tumors. An immunohistochemical study. F Cutan Pathol 1995;22:149-53.

15 Choundhry R, Hodgin MB, Van der Kwast TH, et al. Localisation of androgen receptors in human skin by immunohistochemistry: implication for the hormonal regulation of hair growth, sebaceous glands and sweat glands. F Endocrinol 1992;133:467-75.

16 Ioffe OB, Alonsozana E, Drachenberg CB, et al. Hormone receptors in anogenital hidradenoma papilliferum (ahp): correlation with morphology and comparison with benign breast proliferations [abstract]. Mod Pathol 1998;11:50A.

17 Eusebi V, Damiani S, Losi L, et al. Apocrine differentiation in breast epithelium. Adv Anat Pathol 1997;4:139-55.

18 Galatica Z. Immunohistochemical analysis of apocrine breast lesions. Consistent over-expression of androgen receptor accompanied by the loss of estrogen and progesterone receptors in apocrine metaplasia and apocrine carcinoma in situ. Pathol Res Pract 1997;

19 Simon KE, Dutcher JP, Runowicz CD, et al. Adenocarcinoma arising in vulvar breast tissue. Cancer 1988;62:2234-8.

20 Donati P, Amantea A. Adenoma of anogenital mammarylike glands. Am f Dermatopathol 1996;18:73-6.

21 Nenci I, Beccati MD, Arslan Pagnini C. Estrogen receptors and post-receptor markers in human breast cancer. Tumori 1978;64:161-74

22 Horwitz KB, McGuire W. Estrogen control of progesterone receptor in human breast cancer. Correlation with nuclear processing of estrogen receptors. F Biol Chem 1978;253. 2223-8. 